\title{
Gene Therapy: A Promising Therapeutic Strategy for Malaria
}

\author{
Raafay Shehzad \\ King's College London, London, United Kingdom
}

ABSTRACT

Malaria is a serious illness caused by the Plasmodium parasite, which places approximately 3.5 billion people at risk. Currently, preventative measures are key in combatting this disease. However, gene therapy is an emerging field that shows promising results for the treatment of malaria, by modifying cells through the delivery of genetic material. Most notable was the discovery of CRISPR-Cas9, which not only allows deleterious mutations to be repaired, but does so with specificity, speed, and simplicity. There are numerous ongoing trials focusing on gene therapy in malaria treatment and prevention. They involve different approaches such as the genetic modification of vector mosquitoes to interfere with malaria transmission, use of CRISPR-Cas9, maternal-effect dominant embryonic arrest, homing endonuclease gene drive systems, and the design of specific Morpholino oligomers to interfere with the expression of parasitic characteristics. Overall, this emerging field shows promising results to treat and prevent not just malaria, but other diseases such as cancer, diabetes, and obesity.

\section{RÉSUMÉ}

Le paludisme est une maladie sévère causée par la parasite Plasmodium, qui met en danger environ 3.5 milliards de personnes. Actuellement, des mesures préventives sont la clé pour combattre cette maladie. Toutefois, la thérapie génique est un domaine émergent qui montre des résultats prometteurs pour le traitement du paludisme, en modifiant des cellules par la livraison de matériel génique. En particulier, il faut reconnaitre la découverte de CRISPR-Cas9, qui permet non seulement la réparation de mutations délétère, mais la fait avec spécificité, rapidité et simplicité. Il y a plusieurs essais en cours concentrant sur la thérapie génique pour le traitement et la prévention du paludisme. Ils impliquent plusieurs approches telles que la modification génique de moustiques vecteurs pour intervenir dans la transmission du paludisme, I'utilisation de CRSIPR- Cas9, I'utilisation du gène Medea (arrêt embryonnaire dominant d'effet maternel), les systèmes de forçage génique par endonucléase de homing, et la conception d'oligomères morpholino spécifiques pour intervenir dans l'expression de caractéristiques parasitiques. En gros, ce domaine émergent montre de résultats prometteurs pour le traitement et la prévention de non seulement le paludisme, mais d'autres maladies telles que le cancer, le diabète et l'obésité.

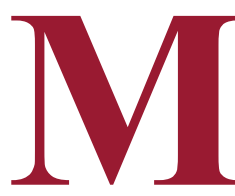

alaria is a severe disease that places almost 3.5 billion people at risk, resulting in approximately 500 million new infections and 473,000 deaths per year $(1,2)$. It is caused by several species of parasitic protozoans of the genus Plasmodium, which are transmitted through the bite of the vector mosquito Anopheles. Malaria symptoms include fever, vomiting, fatigue, and headaches (3). However, in severe cases, jaundice, seizures, coma or even death have been observed. Symptoms usually arise within 10-15 days of being bitten, and if left untreated can reoccur months later. Malaria primarily affects children under the age of 5 and has a significant impact on pregnant women (2). Thus, it is imperative that an effective vaccine is developed, as nearly half the world's population is at risk. This article will serve as a review of the current gene therapy strategies that have been developed and tested for combatting malaria.

\section{LIFE CYCLE}

The development of a malaria vaccine is difficult, in large part due to the complex life cycle of the malaria parasite (2). Transmission of malaria begins when malaria-infected Anopheles mosquitoes bite and inject Plasmodium sporozoites into the human bloodstream. These sporozoites then travel to the liver and infect hepatocytes, thereby initiating the hepatic stage of the parasitic life cycle (4). Subsequently, the sporozoites grow and differentiate into schizonts, which contain a multitude of haploid merozoites. The merozoites leave the hepatocytes and enter the bloodstream, where they

Keywords: Gene therapy; Malaria; Gene editing; CRISPR 
invade erythrocytes and begin the asexual erythrocytic stage of the parasitic life cycle (5). For certain species such as $P$. vivax and $P$. ovale, however, the hepatic stage of the life cycle does not involve the development of schizonts from the sporozoites (6).

Inside the erythrocytes, the merozoites mature into erythrocytic schizonts. At full maturity, the erythrocyte ruptures, releasing new merozoites that can then invade additional erythrocytes. This cycle repeats every few days, resulting in a multitude of malaria-infected erythrocytes (5). In some cases, the merozoites can leave the asexual replication cycle, and develop into sexual male and female gametocytes. Initially, gametocytes remain in the bone marrow and some other organs, but after maturation, gametocytes typically circulate within the bloodstream. Malaria is transmitted to the mosquito after it bites an infected human, ingests the gametocytes, and the erythrocytes within the midgut rupture and release gametocytes, which further develop into mature gametes and later fuse to form a diploid zygote. After further maturation, the zygote develops into a motile ookinete and burrows through the midgut to form an oocyst on the opposite side. After 8-15 days of maturation the oocyst ruptures, releasing thousands of sporozoites that may migrate to the mosquito's salivary glands. Some malaria parasites can form hypnozoites, which can remain dormant for extended periods of time, causing reoccurrences of symptoms weeks to months later (7).

\section{CURRENT THERAPEUTIC OPTIONS}

The treatment of malaria depends upon which Plasmodium species has caused the infection. Almost $75 \%$ of all reported malaria cases are caused by $P$. falciparum, which is responsible for the most severe cases. Most non- $P$. falciparum cases are caused by $P$. vivax, and a few cases are reported by the other two species $P$. ovale and $P$. malariae. They are typically treated with chloroquine $(10 \mathrm{mg} / \mathrm{kg})$ for a period of $24-48 \mathrm{~h}$. The problem with treatment of $P$. falciparum is that the parasite is resistant to many antimalarial drugs available on the market, resulting in increased severity of $P$. falciparum infections. Its treatment consists of intravenous or intramuscular therapy of artesunate $(4 \mathrm{mg} / \mathrm{kg})$ and amodiaquine $(10 \mathrm{mg} / \mathrm{kg})$ for 3 days (8). Complete protection can be achieved in humans via immunization with live, irradiation-attenuated sporozoites; however, this is impractical for widespread use (9).

\section{CURRENT APPROACHES TO GENE THERAPY}

There are numerous ongoing trials focusing on gene therapy for malaria treatment and prevention. They involve different approaches, such as the development of a genetic vaccination using plasmid DNA (2); genetically modifying vector mosquitoes to interfere with malaria transmission (intended to prevent parasite development and lower transmission) (9), Cas9-mediated modification of the Anopheles mosquito to breed within a mosquito population that does not support the malaria parasite (10), genome editing of Plasmodium sp. to remove its parasitic effects in humans (11), and designing specific morpholino oligomers to interfere with the expression of parasitic characteristics (12). Malaria treatment will require vector-control strategies that are self-sustaining and unaffected by the migration of infected humans or mosquitoes, and must also be complemented by other therapeutic means due to the continuous evolution of resistant Plasmodium strains.

\section{Plasmid DNA Vaccination}

Developing a vaccination for malaria has proven difficult, given that the Plasmodium parasite transitions through various stages over the course of its life cycle. A successful malaria vaccination therefore requires multiple components, which can target each stage of the parasite's life cycle (2). A nucleic acid-based vaccination shows promise as it can be genetically modified to induce the expression of the required antigens for each stage in the life cycle. Researchers have shown that in a mouse model, intramuscular injection of a DNA-based vaccine increased serum levels of antibodies specific to the malaria parasite, thus providing protective immunity (13). However, another phase 1 clinical trial conducted in 2016 for the EP1300 DNA vaccine against $P$. falciparum was not deemed fit for further progression due to several safety issues, where subjects experienced spontaneous adverse effects and reported local reactogenicity (14).

\section{Vectored Immunoprophylaxis}

Immunoprophylaxis has been pursued in malaria prevention for over 50 years. Significant investments in this field have led to the development of the RTS,S vaccine, which contains a fusion protein derived from different domains of the circumsporozoite protein (CSP), an antigen present on the surface of the malaria parasite during its infective stages. In a phase 3 trial conducted in 2012 (15), this vaccine provided modest protection against the $P$. falciparum malaria parasite in infants when critical serum concentrations were reached. The World Health Organization (WHO) is presently coordinating a pilot program to evaluate the feasibility of routine administration of this vaccine in African countries (16). 
Developments in the field of gene therapy have allowed for a new strategy involving vectored immunoprophylaxis (VIP), which involves the delivery of genes encoding targeted neutralizing antibodies to human cells (1). This approach utilizes adeno-associated virus-based vectors (AAV), which do not integrate into the human genome and allow for the expression of antibodies for months to years. Genetic components of mouse antibodies were inserted into the human IgG gene to develop AAV encoding antibodies targeted against the P. falciparum circumsporozoite protein (17). These tests were carried out in mice to validate the approach. The results show that intramuscular injection of these AAV can neutralize malaria sporozoites and can provide protection from mosquitotransmitted malaria. This approach is currently not validated for human use. However, after the approval an AAV vector to treat Familia Lipoprotein Lipases deficiency in Europe, further experiments are being carried out in non-human primates to determine VIP protocols for dosage and injection sites (18).

\section{Genetic Manipulation of Mosquito Vector Competence}

Plasmodium transmission is dependent upon the Anopheles mosquito. Reducing or eliminating malaria transmission can be accomplished by preventing the mosquito from supporting parasite development. A probable solution would be to genetically modify the expression of effector genes within the Anopheles mosquito midgut, in order to express products that inhibit parasite development. For example, Anopheles stephensi mosquitoes were genetically engineered to express the SM1 peptide (9), which binds to an ookinete receptor on the luminal surface of the midgut epithelium and prevents ookinete midgut invasion. These genetically modified mosquitoes were impaired in their ability to transmit the malaria parasite. This proof of concept shows that Plasmodium transmission can be reduced or eliminated via genetic modification of the vector mosquito. However, this method faces many challenges namely the translation of these effector gene modifications into wild mosquitoes as releasing transgenic mosquitoes into the wild is simply not enough. One would have to give these transgenic mosquitoes a competitive advantage over wild mosquitoes for gene transmission to be effective. Approaches such as MEDEA (maternal-effect dominant embryonic arrest) and HEG (homing endonuclease gene) are two proposed approaches utilizing transposable elements.

The MEDEA drive system has shown positive results in the Drosophila model system. It involves linking the effector gene to a toxin that inactivates an essential maternal gene required for embryonic development (9). Transferring this technique into mosquitoes is not yet feasible as the technology required does not exist. However, with time this approach may be seen in the practical field.

The HEG drive system encodes highly specific endonucleases that contain recognition sequences (19). Mosquitoes carrying the HEG can mate with wild mosquitoes and their resulting offspring will have an increased likelihood of inheriting the drive gene, eventually resulting in HEG expression within the entire population. HEGs have shown to induce chromosomal cleavage and gene conversion, and inhibit parasite development within the progeny of these transgenic mosquitoes (19).

\section{RNA Interference}

RNA interference (RNAi), involving the use of non-coding RNA fragments paired with specific molecular machinery to silence genes, has become a widespread mechanism for gene regulation. This type of epigenetic regulation is heritable and present in a variety of eukaryotes, but its existence in Plasmodium is debated (20). Initiation of this mechanism begins with the introduction of double stranded RNA (dsRNA) to target a specific gene product. The dsRNA is cleaved into short interfering RNAs (siRNAs) of 21-23 nucleotides in length by the enzyme Dicer, a cytoplasmic dsRNA-specific RNaselll endonuclease. The siRNAs are unwound into two single stranded siRNAs, where one strand (the passenger strand) is degraded, and the other (the guide strand) becomes associated with the RNA-induced Silencing Complex (RISC). Once associated, the guide strand helps RISC to locate a complementary mRNA strand. Once found, the mRNA is cleaved by the enzyme Slicer, a protein from the Argonaute family that resides within the RISC (21). RNAi is therefore a potent and extremely specific technique that allows for reverse genetic experimentation and provides a time-efficient means to determine gene function.

Application of RNAi to malaria is still in early stages of research. However, studies have shown successful growth inhibition via RNAi $(22,23)$.In one study conducted by McRobertandMcConkey (24), RNAi was used to target the gene encoding dihydroorotate dehydrogenase (DHODH), which is an enzyme involved in pyrimidine synthesis. Unlike most organisms, malaria parasites utilize de novo pyrimidine synthesis; therefore, the disruption of DHODH should inhibit parasite growth. In the same study, RNAi was applied to the gene for chorismate synthase (CS), which is involved in the synthesis of chorismate, a precursor in folate synthesis via the shikimate pathway. Parasite growth 
was inhibited from 50 to $90 \%$ via RNAi, suggesting that $\mathrm{DHODH}$ and $\mathrm{CS}$ are valid chemotherapeutic targets for gene silencing to limit Plasmodium growth. This application of RNAi provides useful insight and lays a strong foundation for developing an effective vaccine and anti-malarial chemotherapeutic strategy.

Research indicates that the introduction of dsRNA into the asexual forms of the malaria parasite could downregulate gene expression (25). However, genomic analysis of Plasmodium confirmed that there are no genes involved with RNAi and no response to dsRNA could be documented (26).

\section{Morpholino Oligomers}

A morpholino oligomer (MO) is a molecular complex used to modify gene expression. MO have identical base pairing characteristics as DNA and RNA, and resist degradation by nucleases while maintaining a neutral charge. They are utilized in gene editing techniques due to their ability to block access of other molecules to small specific sequences of roughly 25 base pairs in size on RNA. In a 2015 study, Yale researchers utilized MO to directly affect gene expression within $P$. falciparum (12). Since this parasite lacks RNAi machinery, a MO-based, RNA-targeting approach is much more appropriate. The basis behind this technique is much like RNAi, without its respective machinery. These mechanisms take place within the cells of the parasite itself, thus utilizing an MO strategy that targets and block certain key genes. In this way, this strategy can potentially be much more effective than an anti-malarial drug. The research undertaken at Yale suggests that $\mathrm{MO}$ can be utilized to weaken the parasite, and can potentially cause resistant strains becoming susceptible to anti-malarial drugs.

\section{CRISPR-Cas9 Gene Drive}

The CRISPR-Cas9 system has allowed users to genetically engineer endonucleases which can target and cleave specific genomic sequences in a more simple fashion than the previous transcription activator-like effector nucleases (TALENs) system. A Cas9 endonuclease is guided to a target DNA site by a complementary guide RNA, where it can specifically edit the DNA sequence, all without the need for complex protein engineering and selection techniques (27). The CRISPR-Cas9 system offers promising opportunities to develop gene drive systems for the control of mosquito vectors of malaria, due to its specificity and flexibility. The use of the CRISPR-Cas9 system for vector control requires specific engineering to diminish the ability of the Anopheles mosquitoes to transmit disease. Developing an endonuclease that produces a mutation in a gene required for female Anopheles fertility or parasite development is promising in preventing the transmission of malaria from mosquito to humans. It is essential that endonuclease homing is confined to germline cells, either before or during gamete formation. This avoids disruption of the wild type allele and allows for the normal development of heterozygous mosquitoes. It also ensures their viability and fertility, which is critical for transmitting the endonuclease to their progeny.

A 2015 study utilized the CRISPR-Cas9 system as a gene drive mechanism in A. gambiae, which is the most common malaria vector mosquito (27). The researchers identified three genes that, when disrupted, expressed a recessive female-sterility phenotype. CRISPR-Cas9 gene drive constructs were inserted into each of these three loci, and the constructs were designed to target and edit each gene. Two classes of mosquitoes were found in the progeny; ones which were homozygous and ones which were heterozygous for the edited genes. The homozygous female mosquitoes were infertile, while the heterozygous female mosquitoes were fertile. Both homozygous and heterozygous males were fertile. A strong gene drive was observed at each of the loci, and the noted transmission rates to progeny were significantly high, ranging from $91.4-99.6 \%$. The study showed that gene knockouts at the three loci were achieved with a high frequency, and the rates of inheritance that were observed provide a solid basis for the development of a gene drive system that shows promise in reducing mosquito populations. The study validates the CRISPR-Cas9 gene drive system as a valuable gene editing tool that will play a vital role in functional genetics in the malaria vector mosquito, due to its suitability for population-modification strategies that incorporate the use of transgenes with expressed phenotypes, such as parasite resistance and female fertility.

A study conducted at UCLA-San Diego (28) successfully engineered a gene drive in fruit flies using the CRISPR-Cas9 gene drive system, which provided beneficial information to the researchers, who then tested the Cas 9 system with $A$. stephensi (10). The researchers used the mutagenic chain reaction (MCR) method with CRISPR-Cas9 to produce mutations in fruit flies, which would then be copied from one chromosome to another, with the intent of producing a homozygous mutation resulting in a loss of function (28). The principle was then applied to mosquitoes, where the genes targeted were involved with malaria resistance. The modified genes were present in the offspring of these mutant mosquitoes, which were also resistant 
to malaria.

Gene drive is a profound technique that, when coupled with the CRISPR-Cas9 system, can modify not only a single organism, but an entire population. In the case of malaria, this technique can be used to produce mosquitoes that are incapable of carrying the malaria parasites, but it can also have unanticipated environmental costs that may be irreversible. As of now, the CRISPR-Cas9 system is limited by the currently available delivery methods, as well as the risk of possible off-target edits that may lead to deleterious mutations. Advancements and computational optimization of the guide RNAs has resulted in significantly more accurate gene editing with minimal off-target modifications. CRISPR gene drive technology is developing at an extremely fast pace with a wide variety of applications. However, the fact remains that it has the potential to alter ecosystems in unpredictable ways.

\section{CONCLUSION}

Malaria is one of the world's most severe diseases, killing nearly one million people every year. Since non-genetic techniques have slowly reached a plateau regarding the effective treatment of malaria, genetic techniques have begun to prevail. While VIP can be a viable treatment option, VIP protocols need to be modified and tested regarding dosage and injection site. HEG gene drive systems do show some success, although the CRISPR-Cas9 system prevails due to its specificity and flexibility regarding its complex engineering and selection techniques. The aforementioned techniques therefore provide a foundation for future research methodologies intended to combat malaria, whether it be through the development of an effective multicomponent vaccination, the introduction of malaria resistant mosquito populations, or by genetically modifying the pathogen itself.

\section{REFERENCES}

1. Rodrigues MM, and Soares IS. Gene-therapy for malaria prevention. Trends Parasitol. 2014 Nov;30(11):511-3

2. Parker $S$, Monteith D, Horton $H$, Hof R, Hernandez P, Vilalta, A, et al. Safety of a GM-CSF adjuvant-plasmid DNA malaria vaccine. Gene Ther. 2001 Jul;8(13):1011-23.

3. Carabello H, King K, Akhtar S, Bentley S. Emergency department management of mosquito-borne illness: malaria, dengue, and west nile virus. Emerg Med Pract. 2014 May;16(5):1-23.

4. Soulard V, Bosson-Vanga $H$, Lorthiois A, Roucher C, Franetich JF, Zanghi $\mathrm{G}$, et al. Plasmodium falciparum full life cycle and plasmodium ovale liver stages in humanized mice. Nat Commun. 2015 Jul;6:7690.

5. Hoffman SL, Vekemans J, Richie TL, Duffy PE. The march toward malaria vaccines. Am J Prev Med. 2015 Dec;49(6 Suppl 4):S319-3.

6. Galinski MR, Meyer EV, Barnwell JW. Plasmodium vivax: modern strategies to study a persistent parasite's life cycle. Adv Parasitol. 2013;81:1-26.

7. Gazzinelli R, Kalantari P, Fitzgerald K, Golenbock, D. Innate sensing of ma- laria parasites. Nat Rev Immunol. 2014 Nov;14(11):744-57.

8. White NJ, Pukrittayakamee S, Hien TT, Faiz MA, Mokuolu OA, Dondorp AM. Malaria. Lancet. 2014 Feb;383(9918):723-35

9. Wang S, and Jacobs-Lorena M. Genetic approaches to interfere with malaria transmission by vector mosquitoes. Trends Biotechnol. 2013 Mar;31(3):18593.

10. Gantz V, Jasinskiene N, Tatarenkova O, Fazekas A, Macias V, Bier E, et al. Highly efficient Cas9-mediated gene drive for population modification of the malaria vector mosquito Anopheles stephensi. Proc Natl Acad Sci U S A. 2015 Dec;112(49):E6736-43.

11. Ghorbal M, Gorman M, Macpherson C, Martins R, Scherf A, Lopez-Rubio J. Genome editing in the human malaria parasite Plasmodium falciparum using the CRISPR-Cas9 system. Nat Biotechnol. 2014 Aug;32(8):819-21.

12. Garg A, Wesolowski D, Alonso D, Deitsch K, Mamoun C, Altman S. Targeting protein translation, RNA splicing, and degradation by morpholino-based conjugates in plasmodium falciparum. Proc Natl Acad Sci U S A. 2015 Sep;112(38):11935-40.

13. Sedegah $M$, Hedstrom $R$, Hobart $P$, Hoffman SL. Protection against malaria by immunization with plasmid DNA encoding circumsporozoite protein. Proc Natl Acad Sci U S A. 1994 Oct;91(21):9866-70.

14. Schwartz L, Brown G, Genton B, Moorthy V. A review of malaria vaccine clinical projects based on the WHO rainbow table. Malar J. 2012 Jan 9;11:11.

15. Agnandji ST, Lell B, Fernandes JF, Abossolo BP, Kabwende AL, Adegnika AA et al. A phase 3 trial of RTS,S/AS01 malaria vaccine in African infants. $N$ Engl J Med. 2012 Dec;367 (24):2284-95.

16. Q\&A on the malaria vaccine implementation programme (MVIP) [Internet]. World Health Organization. World Health Organization; 2018 [cited 2018Nov3]. Available from: https://www.who.int/malaria/media/malariavaccine-implementation-qa/en/

17. Deal C, Balazs AB, Espinosa DA, Zavala F, Baltimore D, Ketner G. Vectored antibody gene delivery protects against Plasmodium falciparum sporozoite challenge in mice. Proc Natl Acad Sci U S A. 2014 Aug;111(34):12528-32.

18. Lee AH, Symington LS, Fidock DA. DNA repair mechanisms and their biological roles in the malaria parasite Plasmodium falciparum. Microbiol Mol Biol Rev. 2014 Sep;78(3):469-86.

19. Windbichler N, Menichelli M, Papathanos P, Thyme S, Li H, Ulge U, et al. A synthetic homing endonuclease-based gene drive system in the human malaria mosquito. Nature. 2011 May;473(7346):212-5.

20. Ipsaro JJ, and Joshua-Tor L. From guide to target: molecular insights into eukaryotic RNAi machinery. Nat Struct Mol Biol. 2015 Jan;22(1):20-8.

21. Baum J, Papenfuss A, Mair G, Janse C, Vlachou D, Waters A, et al. Molecular genetics and comparative genomics reveal RNAi is not functional in malaria parasites. Nucleic Acids Res. 2009 Jun;37(11):3788-98.

22. Cottrell T, and Doering T. Silence of the strands: RNA interference in eukaryotic pathogens. Trends Microbiol. 2003 Jan;11(1):37-43.

23. Sriwilaijiaroen N, Boonma S, Attasart P, Pothikasikorn J, Panyim S, Noonpakdee W. Inhibition of Plasmodium falciparum proliferation in vitro by double-stranded RNA directed against malaria histone deacetylase. Biochem Biophys Res Commun. 2009 Apr;381(2):144-7.

24. McRobert L, McConkey G. RNA interference (RNAi) inhibits growth of Plasmodium falciparum. Mol Biochem Parasitol. 2002 Feb;119(2):273-8.

25. Kolev N, Tschudi C, Ullu E. RNA interference in protozoan parasites: achievements and challenges. Eukaryot Cell. 2011 Sep;10(9):1156-63.

26. Angaji S, Hedayati S, Poor R, Madani S, Poor S, Panahi, S. Application of RNA interference in treating human diseases. J Genet. 2010 Dec;89(4):527-37.

27. Hammond A, Galizi R, Kyrou K, Simoni A, Siniscalchi C, Katsanos D, et al. A CRISPR-Cas9 gene drive system targeting female reproduction in the malaria mosquito vector Anopheles gambiae. Nat Biotechnol. 2016 Jan;34(1):78-83.

28. Gantz VM, Bier E. The mutagenic chain reaction: A method for converting heterozygous to homozygous mutations. Science. 2015 Apr;348(6233):4424. 